ALLEX JARDIM DA FonseCA ${ }^{1}$

RaISA SARON WANDERLEY MURARI ${ }^{2}$

Iane Santana Moraes ${ }^{3}$

ROBLEDO FONSECA ROCHA ${ }^{4}$

Luiz Carlos de Lima Ferreira ${ }^{5}$

\title{
Acurácia dos exames citológicos cervicovaginais em Estado de elevada incidência de câncer de colo de útero
}

\author{
Validity of cervicovaginal cytology in a Brazilian State \\ with high incidence rate of cervical cancer
}

Artigo Original

\begin{abstract}
Palavras-chave
Citologia cervical

Neoplasias do colo do útero/diagnóstico Neoplasias do colo do útero/epidemiologia

Keywords

Cervical cytology

Uterine cervical neoplasms/diagnosis Uterine cervical neoplasms/epidemiology
\end{abstract}

\section{Resumo}

OBJETIVO: Avaliar a acurácia da citologia cervicovaginal realizada no principal laboratório do Estado de Roraima, por concordância entre avaliadores (monitoramento externo) e com resultados histopatológicos. MÉTODOS: Lâminas de 100 mulheres, de uma amostra de conveniência de base populacional, foram avaliadas pelo LAPER, principal laboratório de referência do Sistema Único de Saúde do Estado de Roraima e reavaliadas por médicos patologistas experts externos ao laboratório. Foram avaliados índice Kappa, sensibilidade e especificidade. $\bigcirc$ estudo foi aprovado pela Coordenação do Laboratório Estadual e pelo Comitê de Ética em Pesquisa da Universidade Federal de Roraima. RESULTADOS: Quanto à prevalência de atipias celulares (relacionadas ao papilomavírus humano), não houve concordância entre resultados emitidos pelo LAPER e pelos patologistas externos $(k=0,21)$. Constatou-se baixa sensibilidade $(28,5 \%)$ e especificidade de $89,2 \%$ para o desempenho do LAPER, com elevada proporção de resultados falsos positivos e falsos negativos. Os laudos citológicos de patologistas externos apresentaram sensibilidade e especificidade maiores (71,4 e 98,9\%, respectivamente), descartando que erros de coleta e coloração possam explicar o baixo desempenho do LAPER. CONCLUSÃO: O baixo desempenho diagnóstico da citologia cervicovaginal pode ser uma barreira para o controle do câncer de colo de útero em Roraima. Ressalta-se a necessidade de capacitação profissional e monitoramento interno e externo em Estados brasileiros com alta incidência de câncer de colo de útero.

\section{Abstract}

PURPOSE: To evaluate the validity of cervicovaginal cytology performed at LAPER, the main Laboratory of Pathology of the State of Roraima, Brazil, by interrater agreement (external monitoring) and agreement with histopathologic results. METHODS: One hundred women were included, a population-based convenience sample. Their cervical cytological exams were evaluated by the laboratory staff and reviewed by expert medical pathologists, external to the laboratory. Cohen's Kappa index, sensitivity and specificity were evaluated. The study was approved by LAPER coordination and Federal University of Roraima Research Ethics Commitee. RESULTS: Regarding the prevalence of human papillomavirus-related atypical, there was no concordance between the results issued by LAPER and by the external pathologists ( $k=0.21$ ). A low sensitivity (28.5\%) and specificity $(89,2 \%)$ was detected for the diagnostic performance of LAPER, with a high proportion of false positive and false negative results. The cytological reports of the external pathologists showed higher sensitivity and specificity 171.4 and $98.9 \%$, respectively), ruling out the possibility that errors related to collection methods and staining would explain the low performance of the laboratory. CONCLUSION: A low diagnostic accuracy of cervicovaginal cytology can be a barrier against the control of cervical cancer in Roraima. We emphasize the need for professional training and internal and external monitoring in Brazilian states with a high incidence of cervical cancer.
Correspondêncio

Allex Jardim da Fonseco Avenida Pedro Teixeira, 25 - Dom Pedro CEP: $69040-000$

Manaus (AM), Brasil

Recebido

$17 / 06 / 2014$

Aceito com modificacọōes

$07 / 07 / 2014$
Fundação de Medicina Tropical Dr. Heitor Vieira Dourado, Universidade do Estado do Amazonas - Manaus (AM); Centro de Ciências da Saúde, Universidade Federal de Roraima - Boa Vista (RO), Brasil.

IPrograma de Pós-graduação em Medicina Tropical, Fundação de Medicina Tropical Dr. Heitor Vieira Dourado, Universidade do Estado do Amazonas - Manaus (AM); Programa de Pós-graduação em Ciências da Saúde, Centro de Ciências da Saúde, Universidade Federal de Roraima - Boa Vista (RO), Brasil.

${ }^{2}$ Hospital Geral de Roraima - Boa Vista (RO), Brasil.

${ }^{3}$ Centro de Ciências da Saúde, Universidade Federal de Roraima - Boa Vista (RO), Brasil.

${ }^{4}$ Departamento de Patologia, Centro de Ciências da Saúde, Universidade Federal de Roraima - Boa Vista (RO), Brasil.

SPrograma de Pós-graduação em Medicina Tropical, Fundação de Medicina Tropical Dr. Heitor Vieira Dourado, Universidade do Estado de Roraima - Manaus (AM), Brasil.

Conflito de interesses: não há. 


\section{Introdução}

A redução na incidência e na mortalidade decorrentes do câncer de colo de útero (CCU) observada globalmente nas últimas quatro décadas não ocorreu de forma homogênea, mas principalmente em países desenvolvidos que conseguiram implementar programas preventivos sólidos de rastreamento populacional baseado em citologia cervicovaginal do colo de útero ${ }^{1}$. Atualmente, $80 \%$ dos casos de CCU ocorrem em países em desenvolvimento econômico, como o Brasil, e correspondem a aproximadamente $15 \%$ de todos os tipos de câncer feminino ${ }^{2}$.

A estratégia governamental brasileira para controle do CCU tem se concentrado no rastreamento de mulheres de 25 a 64 anos, com periodicidade de 3 anos, após 2 exames anuais consecutivos com resultado normal ${ }^{3}$. Entretanto, a persistência de elevadas taxas de incidência e mortalidade por CCU no país aponta para uma ainda insuficiente efetividade dos programas. No Brasil, onde atualmente o CCU é a principal causa de morte por câncer em mulheres jovens (até 35 anos) ${ }^{1}$, são estimados quase 20 mil novos casos (18/100.000) em 2014 .

Muitos fatores podem explicar o sucesso parcial dos programas de rastreamento, como baixa cobertura populacional da estratégia preventiva, falhas no acompanhamento das mulheres com anormalidades citológicas, adoção de condutas terapêuticas inadequadas, entre outros 5 . Porém, um fator decisivo é a vulnerabilidade da técnica do Papanicolaou a erros de coleta e subjetividade da leitura das lâminas ${ }^{6}$. Apesar dos esforços do Ministério da Saúde para padronização da leitura das lâminas ${ }^{7}$, há evidências de que a sensibilidade e a especificidade da citologia cervicovaginal variam de acordo com a região analisada, podendo afetar adversamente os resultados de estratégia preventiva ${ }^{8,9}$.

A região amazônica caracteriza-se por elevada incidência de CCU, sendo a única região brasileira onde o CCU é mais incidente que o câncer de mama ${ }^{4}$. Em especial, o Estado de Roraima destaca-se por apresentar a mais alta taxa de incidência de CCU do país. Um estudo de base populaciona ${ }^{10}$ revelou elevada taxa de incidência bruta dessa doença em 2010 em Roraima (46,2/100.000), superando as estimativas do Instituto Nacional do Câncer (INCA), incidência comparável à de países subdesenvolvidos de baixa renda. Entretanto, há mais de uma década, o monitoramento das ações programáticas de controle do CCU aponta elevada cobertura do teste de citologia cervicovaginal no Estado de Roraima ( $>85 \%$ da população-alvo) ${ }^{7}$. Além disso, em 2008, o IBGE divulgou o resultado da Pesquisa Nacional por Amostra de Domicílios (PNAD) ${ }^{11}$ referente ao acesso e à utilização de serviços de saúde, entre outros dados. Nesse inquérito domiciliar, 72,7\% das mulheres de Roraima (entre 25 e 60 anos) afirmaram ter sido submetidas ao teste de Papanicolaou, ao passo que para a região Norte essa taxa foi de $76,9 \%$ e para o Brasil, de $78,4 \%$. Um inquérito telefônico ${ }^{12}$ realizado pelo Ministério da Saúde divulgou que 79,6\% das mulheres de Roraima informaram ter realizado o exame nos últimos três anos, uma adesão acima da média nacional.

Considerando que a Organização Mundial da Saúde (OMS) recomenda uma cobertura populacional de aproximadamente $80 \%$ para controlar a morbidade e a mortalidade por CCU em uma região, as evidências sugerem que falhas em outra etapa da estratégia preventiva estejam implicadas no sucesso parcial do programa preventivo em Roraima. Não existem informações sobre a acurácia do teste de citologia cervicovaginal em Roraima (Região Norte do Brasil). O objetivo deste estudo é avaliar a validade do teste preventivo do CCU realizado na rede do Sistema Único de Saúde (SUS) do Estado de Roraima.

\section{Métodos}

O estudo foi desenhado para avaliar a concordância de resultados de exames histopatológicos do colo de útero (padrão ouro) e exames de citologia cervicovaginal realizados pelo principal laboratório da rede SUS do Estado de Roraima e por avaliadores independentes (médicos patologistas externos), com uma amostra de base populacional, que faz parte de uma linha de pesquisa que investiga a magnitude da infecção por papilomavírus humano (HPV) em populações nativas do Estado de Roraima. O projeto foi previamente aprovado pela Comissão Nacional de Ética em Pesquisa (Protocolo 16.800; Parecer 325/2012). O presente estudo também foi aprovado pelo Comitê de Ética em Pesquisa da Universidade Federal de Roraima - UFRR (Parecer 686.700/2014) e pela Coordenação do Laboratório de Patologia do Estado de Roraima (LAPER). A utilização de Termo de Consentimento Livre e Esclarecido foi dispensada em razão da natureza secundária dos dados analisados.

A amostra deste estudo - uma amostra de conveniência - foi extraída de um estudo original de coorte, iniciado em 2013, que avalia a incidência de lesões invasivas e precursoras de CCU e de infecção por HPV pela técnica de PCR com sequenciamento genético. Foram selecionadas aleatoriamente 100 mulheres cujo exame foi positivo para DNA de HPV (qualquer genótipo). Todas haviam sido avaliadas inicialmente por citologia cervicovaginal, reavaliadas por colposcopia e submetidas à biópsia do colo de útero por cirurgia de alta frequência.

Primeiramente, as lâminas de citologia cervicovaginal foram avaliadas por médicos patologistas seniores externos ao laboratório onde foram inicialmente avaliadas (professores universitários de patologia e experts em citopatologia). Posteriormente, as mesmas lâminas foram entregues e reavaliadas, cegamente, no LAPER, localizado 
na capital Boa Vista, atualmente responsável pelos laudos de exames de citologia do colo de útero da rede SUS do Estado de Roraima, servindo a Unidades de Saúde Básica, Centros de Referência em Saúde da Mulher e Hospitais Terciários do Estado. As amostras histológicas do colo de útero, coletadas em momento posterior ao da citologia, foram também avaliadas pelos patologistas externos (também cegamente), e os resultados de histopatologia foram analisados como padrão ouro.

No estudo original, para obtenção de amostras da ectocérvice uterina e de fundo de saco vaginal foi utilizada a Espátula de Ayres. A coleta de material representativo do canal endocervical foi realizada com o uso da escova endocervical. As amostras foram distendidas em lâminas de vidro, duplamente identificadas. $\mathrm{O}$ material estendido na lâmina foi imediatamente borrifado com solução fixadora $\left(V_{\text {Vagispec }}^{\circledR}\right.$, Adlin Plástico Ltda., Santa Catarina, Brasil), constituída por propilenoglicol e álcool absoluto, armazenado e transportado para o LAPER. Nesse laboratório, as lâminas foram coradas segundo técnica tradicional, conforme recomendado por diretrizes brasileiras ${ }^{3}$, que consiste de imersões das lâminas consecutivamente em álcool etílico $99,5^{\circ} \mathrm{GL}$, corante hematoxilina, corante Orange, corante EA-50 e xilol.

A leitura das lâminas foi realizada segundo as diretrizes brasileiras para o rastreamento do CCU e a nomenclatura brasileira para laudos citológicos cervicovaginais ${ }^{13}$. Para fins da pesquisa, três aspectos dos resultados de cada exame foram analisados, a saber: (1) adequabilidade do material da lâmina; (2) alterações celulares benignas; (3) atipias celulares. Os resultados de atipias celulares foram classificados para fins de pesquisa em: (a) normal; (b) lesão intraepitelial de baixo grau (do inglês, LSIL); (c) lesão intraepitelial de alto grau (do inglês, HSIL); (d) carcinoma invasivo; (e) atipia celular escamosa de significado indeterminado (do inglês, ASC-US).

\section{Análise de dados}

Prevalência de atipias citológicas e/ou alterações benignas foi definida como número de exames alterados dividido pelo número de exames coletados. Para comparação entre proporções, foi utilizado o teste do $\chi^{2}$ com correção de Yates. Quando não aplicável (amostras pequenas), foi utilizado o teste exato de Fischer. O nível de significância considerado foi de $5 \%$. Foram calculadas a sensibilidade, a especificidade e os valores preditivos positivos e negativos da leitura dos exames citológicos realizados no LAPER e pelos patologistas externos. Quanto à avaliação de concordância entre avaliadores, foi utilizado o método de Kappa de Cohen. Foi considerada ausência de concordância para valores de Kappa $(\kappa)$ menores de 0,2 ; concordância sofrível entre 0,2 e 0,4 ; fraca entre 0,4 e 0,6 ; moderada entre 0,6 e 0,8 ; e elevada se $>0,8$. As análises estatísticas foram realizadas utilizando o software EpiInfo ${ }^{\circledR} 7.0$ versão para Windows (CDC, Atlanta, US).

\section{Resultados}

Os resultados citológicos emitidos pelos patologistas externos e pelo LAPER concordaram quanto à elevada prevalência de lâminas com amostras satisfatórias (98 e $100 \%$, respectivamente, $\kappa=0,9$ ). Duas lâminas foram consideradas de qualidade insatisfatória pelos patologistas externos, ambas por apresentarem $>75 \%$ de células inflamatórias (neutrofílico).

Quanto às alterações benignas, apesar de nenhum exame ter sido laudado como normal pelo LAPER, mais de um quarto das lâminas (27\%) foi interpretado como normal pelos patologistas, e não houve concordância entre os avaliadores nesse aspecto $(\kappa=0,3)$. A prevalência de resultado com inflamação foi significativamente maior segundo avaliação dos profissionais do laboratório quando comparada à dos patologistas externos (94 versus 67\%, respectivamente; $\mathrm{p}=0,01 ; \kappa=0,1)$. A Tabela 1 compara as prevalências de resultados do LAPER, dos patologistas externos, atribui graus de concordâncias e os compara com o resultado histopatológico.

Em relação ao diagnóstico de atipias celulares, exames normais foram mais comuns quando laudados pelos patologistas externos do que pelo LAPER (91 versus $78 \%$, respectivamente; $\mathrm{p}=0,02 ; \kappa=0,3)$. A prevalência de alterações atípicas (de qualquer grau) sugestivas de infecção por HPV relatada pelo laboratório foi mais que o triplo quando comparada à leitura por patologistas externos ( 5 versus $12 \%$, respectivamente; $\mathrm{p}=0,03$ ). Observou-se baixíssima ou nenhuma concordância entre avaliadores nos quesitos de atipias celulares $(\kappa=0,2)$.

Ao avaliar a correlação entre os laudos citológicos emitidos pelo LAPER e os resultados histopatológicos, observou-se fraca concordância para o diagnóstico normal (ausência de atipia) $(\kappa=0,4)$ e baixíssima concordância (ou nenhuma) para o diagnóstico de LSIL e HSIL ( $\kappa=0,25$ e $\kappa=0,05$; respectivamente). Quando avaliada a correlação entre os laudos citológicos dos patologistas externos e os resultados histopatológicos, observou-se elevada concordância para o diagnóstico normal $(\kappa=0,8)$, assim como para o diagnóstico de LSIL $(\kappa=0,8)$ e de $\operatorname{HSIL}(\kappa=1,0)$.

Considerando o laudo histopatológico como padrão ouro para detecção de atipia celular sugestiva de HPV, a sensibilidade do exame citológico realizado pelo LAPER quanto à presença de lesão intraepitelial de qualquer grau (não ASCUS) foi de apenas 28,5\%, e a especificidade foi de $89,2 \%$ (Tabela 2). Nesse cenário, a avaliação realizada pelos patologistas externos apresentou desempenho substancialmente melhor, sensibilidade de $71,4 \%$ e especificidade de $98,8 \%$. $\mathrm{O}$ valor preditivo positivo (VPP) dos laudos emitidos pelo 
Tabela 1. Correlação entre avaliadores da amostra citológica do colo de útero e o diagnóstico histopatológico (padrão ouro)

\begin{tabular}{|c|c|c|c|c|c|c|}
\hline Resultado & $\begin{array}{l}\text { Diagnóstico histopatológico } \\
\text { (padrão ouro) } \\
\text { (\%) }\end{array}$ & $\begin{array}{l}\text { Interpretação } \\
\text { citologia do LAPER } \\
\text { (\%) }\end{array}$ & $\begin{array}{l}\text { Índice Kappa } \\
\text { (LAPER x } \\
\text { padrão ouro) }\end{array}$ & $\begin{array}{l}\text { Interpretação citologia } \\
\text { de patologistas externos } \\
(\%)\end{array}$ & $\begin{array}{l}\text { Índice Kappa } \\
\text { (patologistas } \\
\text { x padrão ouro) }\end{array}$ & $\begin{array}{c}\text { Índice Kappac } \\
\text { (LAPER } x \\
\text { patologistas) }\end{array}$ \\
\hline \multicolumn{7}{|l|}{ Adequabilidade da amostra cifológica } \\
\hline Insatisfatória & - & 0 & - & 2 & - & \\
\hline \multicolumn{7}{|l|}{ Alterações citológicas benignas } \\
\hline Presença de atrofia & - & 6 & - & 6 & - & 0,6 \\
\hline \multicolumn{7}{|l|}{ Atipias celulares } \\
\hline Normal & 93 & 78 & 0,5 & 91 & 0,8 & 0,3 \\
\hline ASC-US & - & 10 & - & 3 & - & 0,1 \\
\hline LSIL & 6 & 6 & 0,2 & 5 & 0,8 & 0,01 \\
\hline
\end{tabular}

a Índice Kappa entre diagnóstico histopatológico e resultado citológico do LAPER; b Índice Kappa entre diagnóstico histopatológico e resultado citológico de patologistas externos; c Indice Kappa entre resultado citológico do LAPER e resultado citológico de patologistas externos; LAPER: Laboratório de Patologia do Estado de Roraima; ASC-US: atipia celular escamosa de significado indeterminado; LSIL: lesão intraepitelial de baixo grau; HSIL: lesão intraepitelial de alto grau.

Tabela 2. Sensibilidade e especificidade do exame citológico por avaliadores quanto à detecção de atipias celulares sugestivas de HPV

\begin{tabular}{|c|c|c|c|}
\hline & Histopatologia - Resultado com atipia & Histopatologia - Resultado sem atipia & \\
\hline \multicolumn{4}{|l|}{ Citologia laboratório rede SUS } \\
\hline Presença de atipia celular & 2 & 10 & $V P P=16,6 \%$ \\
\hline \multirow[t]{2}{*}{ Exame normal } & 5 & 83 & VPN=94,3\% \\
\hline & Sensibilidade=28,5\% & Especificidade $=89,2 \%$ & \\
\hline \multicolumn{4}{|l|}{ Citologia patologistas externos } \\
\hline Presença de atipia celular & 5 & 1 & VPP $=83,3 \%$ \\
\hline Exame normal & Sensibilidade $=71,4 \%$ & Especificidade $=98,9 \%$ & \\
\hline
\end{tabular}

SUS: Sistema Único de Saúde; VPP: valor preditivo positivo; VPN: valor preditivo negativo.

LAPER com diagnóstico positivo para lesão intraepitelial foi de apenas $16,6 \%$, ao passo que o valor preditivo negativo (VPN) foi $94,3 \%$. Observou-se elevada proporção de falsos positivos (83\%) nessa análise. A acurácia diagnóstica do teste realizado pelo LAPER foi de $24,9 \%$.

\section{Discussão}

Desde a implementação pelo governo brasileiro de medidas para ampliação do acesso da população ao exame de rastreamento do CCU, os citotécnicos têm sido os profissionais de triagem na maioria dos laboratórios públicos, realizando a leitura inicial de $100 \%$ das lâminas e encaminhando os exames alterados (usualmente 10 a 30\%) para verificação por médico patologista ou médicos com especialização em citopatologia ${ }^{14}$. Por ser um teste observador dependente, a acurácia diagnóstica da citologia cervicovaginal é fortemente influenciada por treinamento e capacitação dos profissionais envolvidos na coleta e na leitura. A principal crítica ao desempenho do teste de Papanicolaou, como exame de rastreamento, reside na elevada proporção de falsos negativos, o que negligencia mulheres portadoras de lesões potencialmente preveníveis ou curáveis9.

Este estudo demonstrou que laudos de citologia cervicovaginal emitidos pelo principal laboratório da rede SUS do Estado de Roraima apresentaram baixa concordância em relação à reavaliação por médicos patologistas experts, bem como em relação ao exame histopatológico. O fato de as mesmas lâminas de citologia terem sido reavaliadas cegamente por médicos patologistas externos, e essas avaliações terem obtido desempenho satisfatório em relação à histopatologia, descarta que erros de coleta ou de coloração das lâminas possam justificar o baixo desempenho diagnóstico do LAPER. Ressalta-se que não houve concordância entre avaliadores em relação à presença de inflamação, um dos critérios mais objetivos da avaliação citológica. 
A baixa sensibilidade observada para detecção de lesões pré-malignas cervicais pelo LAPER contraria os princípios de elegibilidade de um teste para triagem de câncer. Para triar uma doença grave e potencialmente curável como o câncer, é fundamental elevada sensibilidade diagnóstica, o que tende a aumentar o valor preditivo negativo, reduzindo a proporção de falsos negativos. Os resultados deste estudo sugerem que uma elevada proporção de mulheres portadoras de lesão intraepitelial cervical, rastreadas na rede SUS do Estado de Roraima para o CCU, recebem resultados citológicos falsos negativos, privando-as de acompanhamento especializado quando ainda assintomáticas e/ou com doença inicial. De fato, evidências apontam que, ainda hoje, a maioria dos casos de CCU é diagnosticada em estádio avançado no Estado de Roraima ${ }^{10}$, apesar de a meta de cobertura populacional de rastreamento ser satisfatória há mais de uma década ${ }^{7}$.

A elevada proporção de falsos positivos também constatada no desempenho diagnóstico do LAPER acarreta outra condição adversa em saúde pública. Pacientes saudáveis, erroneamente apontadas como portadoras de lesões pré-malignas, tendem a ser encaminhadas para Centros de Referência de Saúde da Mulher para prosseguir a investigação com exames adicionais, como colposcopia e biópsia, gerando filas de espera e gastos desnecessários e inúteis. Em situações de escassez de recurso, todos os esforços deveriam ser focados na alocação mais eficiente possível dos recursos disponíveis na sociedade, para que possam ser direcionados para as mulheres que realmente se beneficiariam de exames e atendimento médico especializado ${ }^{15}$.

Este estudo possui limitações. Em razão de a prevalência do desfecho (citologia alterada) ter sido baixa, fica implícita menor confiabilidade dos resultados positivos em decorrência da maior probabilidade de falsos positivos. Isso não invalida o uso do valor preditivo positivo como um indicador de qualidade do exame citológico, porém este deve ser analisado de forma conservadora. Entretanto, a sensibilidade e a especificidade são propriedades exclusivas do teste e não sofrem variação com a prevalência do evento estudado.

Países e regiões que obtiveram sucesso no controle do CCU minimizaram as falhas de desempenho da citologia cervicovaginal por meio da busca constante por qualidade diagnóstica em seus laboratórios ${ }^{16}$.

A principal contribuição deste estudo foi ratificar a observação de como o teste de citologia cervicovaginal é dependente da qualificação e da categoria dos profissionais envolvidos na leitura. Ressalta-se a importância do monitoramento interno e externo de qualidade dos laudos citológicos emitidos para a avaliação do desempenho dos laboratórios integrantes do SUS em regiões de elevada incidência de CCU. Estudos demonstram que a proporção de resultados falsos negativos diminui drasticamente em laboratórios submetidos à avaliação sistemática de qualidade, sendo o monitoramento externo o mais empregado ${ }^{17,18}$.

\section{Referências}

1. WHO/ICO Information Centre on HPV and Cervical Cancer (HPV Information Centre) [Internet]. Summary report on HPV and cervical cancer statistics in Brazil. 2007 [citado 2008 maio 13]. Disponível em: <http://www.hu.ufsc.br/projeto_hpv/OMS\%20 HPV\%20BRAZIL.pdf>

2. Ferlay J, Shin HR, Bray F, Forman D, Mathers C, Parkin DM. Estimates of worldwide burden of cancer in 2008: GLOBOCAN 2008. Int J Cancer. 2010;127(12):2893-917.

3. Brasil. Ministério da Saúde. Instituto Nacional de Câncer. Coordenação Geral de Ações Estratégicas. Divisão de Apoio à Rede de Atenção Oncológica. Diretrizes brasileiras para o rastreamento do câncer do colo de útero. Rio de Janeiro: INCA; 2011.

4. Brasil. Ministério da Saúde. Instituto Nacional de Câncer José Alencar Gomes da Silva. Coordenação de Prevenção e Vigilância [Internet]. Estimativa 2014: incidência de câncer no Brasil. Rio de Janeiro: INCA; 2014 [citado 2014 jun 2]. Disponível em: <http:// www.inca.gov.br/estimativa/2014/estimativa-24042014.pdf>

5. Novaes $H M$, Braga $P E$, Schout $D$. Fatores associados à realização de exames preventivos para câncer nas mulheres brasileiras, PNAD 2003. Ciênc Saúde Coletiva. 2006; 11 (4):1023-35.

6. Girianelli VR, Thuler LC, Szklo M, Donato A, Zardo LM, Lozana JA, et al. [Comparison of hybrid capture II, liquid based cytology and Pap test for the early detection of the cervical cancer in Rio de Janeiro, Brazil]. Rev Bras Cancerol. 2004;50(3):225-6. Portuguese.
7. Brasil. Ministério da Saúde. Instituto Nacional de Câncer. Monitoramento das ações de controle de câncer de colo de útero e mama. Bol Inf Detecção Precoce [Internet]. 2011 [citado 2012 ago 8];2(3). Disponível em: <http://wwwl inca.gov.br/inca/ Arquivos/4a_prova.pdf>

8. Gontijo RC, Derchain SF, Montemor EB, Sarian LO, Serra MM, Zeferino LC, et al. Citologia oncológica, captura de híbridos II e inspeção visual no rastreamento de lesões cervicais. Cad Saúde Pública. 2005;21(1):141-9.

9. Pinho AA, Mattos MC. Validade da citologia cervicovaginal na detecção de lesões pré-neoplásicas e neoplásicas de colo de útero. J Bras Patol Med Lab. 2002;38(3):225-31.

10. Fonseca AJ, Ferreira LP, Dalla-Benetta AC, Roldan CN, Ferreira ML. [Epidemiology and economic impact of cervical cancer in Roraima, a Northern state of Brazil: the public health system perspective]. Rev Bras Ginecol Obstet. 2010;32(8):386-92. Portuguese.

11. Brasil. Ministério do Planejamento [Internet]. Pesquisa Nacional por Amostra de Domicílio: PNAD 2008. Um panorama da saúde no Brasil. Acesso e utilização dos serviços, condições de saúde e fatores de risco e proteção à saúde 2008. Brasília (DF): Ministério da Saúde; 2008 [citado 2014 mar 17]. Disponível em: <http:// biblioteca.ibge.gov.br/visualizacao/monografias/GEBIS\%20 $-\% 20 R J /$ panorama.pdf> 
12. Brasil. Ministério da Saúde. Secretaria de Vigilância em Saúde [Internet]. Vigitel Brasil 2012: vigilância de fatores de risco e proteção para doenças crônicas por inquérito telefônico. Brasília (DF): Ministério da Saúde; 2013 [citado 2014 mar 17]. Disponível em: <http://www.sbpt.org.br/downloads/arquivos/ vigitel_2012.pdf>

13. Brasil. Ministério da Saúde. Instituto Nacional de Câncer José Alencar Gomes da Silva [Internet]. Nomenclatura brasileira para laudos citopatológicos cervicais. 3a ed. Rio de Janeiro: INCA; 2012 [citado 2014 mar 17]. Disponível em: <http://wwwl inca. gov.br/inca/Arquivos/nomenclatura_laudo_cervical.pdf>

14. Thuler LC, Zardo LM, Zeferino LC. Perfil dos laboratórios de citopatologia do Sistema Único de Saúde. J Bras Patol Med Lab. 2007;43(2):103-14.
15. Caetano R, Vianna CM, Thuler LC, Girianelli VR. [Cost-effectiveness of the early diagnosis of cervical cancer in Brazil]. Physis. 2006; 16(1):99-1 18. Portuguese.

16. Crothers BA, Jones BA, Cahill LA, Moriarty AT, Mody DR, Tench WD, et al. Quality improvement opportunities in gynecologic cytologichistologic correlations: findings from the College of American Pathologists Gynecologic Cytopathology Quality Consensus Conference working group 4. Arch Pathol Lab Med. 2013;137(2):199-213.

17. Amaral RG, Santos SH, Catharino JM, Silva LC, Westin MC, Cotta $A C$, et al. Revisão rápida de esfregaços cervicais como método de garantia interna de qualidade. J Bras Patol Med Lab. 2003;39(2):151-5.

18. Tavares SB, Amaral RG, Manrique EJ, Sousa NL, Albuquerque ZB, Zeferino LC. Controle da qualidade em citopatologia cervical: revisão de literatura. Rev Bras Cancerol. 2007;53(3):355-64. 